\title{
THE RESPONSE MECHANISM IN ASCIDIANS
}

\author{
By Graham Hoyle \\ Department of Zoology, University College, London
}

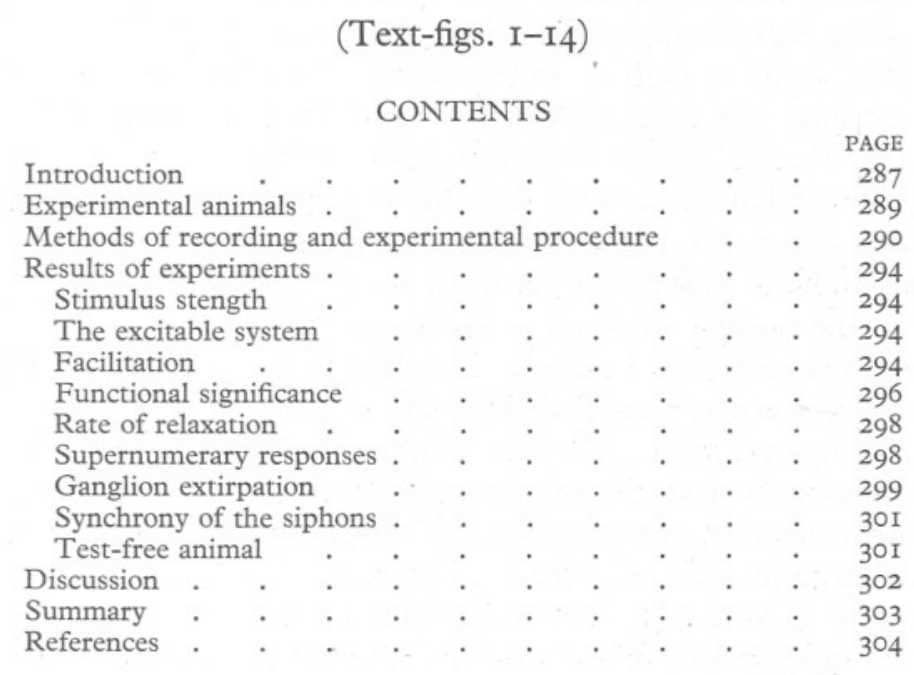

\section{INTRODUCTION}

The responses of tunicates to stimulation have been studied principally by Jordan (I908), Polimanti (I9II), Kinoshita (I9I0), Hecht (I9I8), and Bacq (1935). Jordan and Hecht established the typical responses to mechanical stimulation. A light touch on the outside of either siphon rim causes that siphon to close, the extent of the closure being proportional to the strength of the stimulus. Stronger stimulation causes closure also of the unstimulated siphon. This closure is accompanied by a shortening of the siphons. Stimulation of the body of the animal also causes closure of both siphons together. When the stimulation is still stronger, either applied to a single siphon or to the whole animal, a violent synchronous contraction of the whole musculature occurs, accompanied by the ejection of water from the branchial and atrial cavities. In addition to these simple responses 'crossed responses' occur. Gentle stimulation of the inside of a siphon-rim results in closure of the opposite siphon without affecting the stimulated one. Further stimulation in the same manner results in violent contraction of the whole animal with water ejection through the open stimulated siphon. In some ascidians there is also a reflex bending of the body to vigorous mechanical stimulation. The latter 
activity depends on the presence of unequally thickened walls of the test and is probably due to this structural peculiarity.

Measurements of the siphon contractions during stimulation were made by Kinoshita and Polimanti, who both used Ciona intestinalis. They studied animals which had settled on pieces of rock, or used a clamp to grip the base of the test. Hooks were passed through the siphon rims and attached to levers writing on kymograph paper. The methods of stimulation employed were: (I) mechanical, touching a siphon-rim with a glass rod; (2) electrical, using induction-coil shocks applied through platinum wires. Neither method permits very accurate control and it seems desirable to re-investigate the responses using the more accurately controlled method of stimulation employed by Pantin (1935a-c) for the analysis of the responses of actinians.

The studies of Kinoshita show that the responses of Ciona become rapidly weaker with successive bursts of stimuli. He used spring levers, thereby giving the siphons unaccustomed work to do against the lever system in addition to the normal work done against the mechanical resistance of the test. The test is very weak in Ciona. He claimed that the latent period of the response to an electrical stimulus was about twice that to a mechanical stimulus ( $0.54 \mathrm{sec}$. compared with $0.28 \mathrm{sec}$.). Polimanti, using a pulley system to transfer movements from the animal to the levers, gave the siphons less work to do. He compared the responses of both siphons simultaneously and obtained a large number of records. The results were not consistent. It is clear from his records: (I) that the heights of the contractions of the two siphons are independent of each other on many occasions; (2) that factors which are not obviously related to the given stimuli affect the contractions. In fact, the whole animal cannot be regarded as a simple mechanical system always responding to similar stimuli in the same way. Uncontrollable factors affect both the height of contraction and the rate of relaxation. Other points also appear during a survey of Polimanti's records. Contraction may not start until after several stimuli have been given, although sometimes the contractions start almost immediately after the beginning of stimulation. There are several possible explanations. The latency may be variable, the electrical stimuli may sometimes be ineffective, or some form of inhibition may be present.

As in sea-anemones (Batham \& Pantin, I950) the responses of sea-squirts to stimulation must be observed against a background of spontaneous activity. Rhythmic activity of the siphons was studied by Hecht (I9I8) using Ascidia atra, and by Yamaguchi (I93I) using Styela clava. Both these authors had to take special precautions to protect their experimental animals from external vibrations by working in isolated laboratories built on either a concrete or stone floor, because ascidians are extremely sensitive to mechanical vibrations. However, even with extreme precautions to protect the animals spontaneous contractions were apparent. The spontaneous activity described by Hecht consisted of body-contractions and siphon closures accompanied by water 
ejection, at intervals of about $5 \mathrm{~min}$. Yamaguchi also described spontaneous activity with $5 \mathrm{~min}$. periodicity but claimed that in filtered sea water the pattern of this activity was changed to a more regular type than that observed in ordinary sea water, in which three or four small contractions alternated with larger ones.

\section{EXPERIMENTAL ANIMALS}

The animal principally chosen for investigation was Phallusia mammillata Cuvier. Some duplicate experiments were also done on Ascidiella aspersa Müller. These were so exactly similar in their responses that records from both species have been chosen for use in this paper to illustrate various salient results. These animals are not as sensitive to vibrations as Ciona and have thick tests. Phallusia is especially endowed in this respect. It is available near the Plymouth Laboratory, both in estuaries, where it is exposed at very low tides, and in the Sound to considerable depths. Specimens from I3 to I $5 \mathrm{~cm}$. long can be obtained without damage to the test. They survive for at least a few days in Plymouth tank sea water under circulation but do not live long when transported to London. The estuarine forms are brownish in colour owing to the presence of pigmented cells in the body-wall and the test. The deeper water forms are milky white. The test is nowhere less than a quarter of an inch in thickness except at the siphon rims. It is quite firm even when the blood is drained out and shows viscous-elastic properties when stretched. Gross alteration of the form of the test takes place fairly rapidly when the animal is removed from the sea water. The original form is recovered on returning to water if the animal is still alive. The mantle, or body-wall is firmly attached at each siphon rim but elsewhere is only loosely attached to the test. Contractions of the body-wall musculature result in the expulsion of water from the branchial and atrial cavities accompanied by siphon-rim closure and withdrawal. No conspicuous movements of the whole animal occur. Hence it is possible to study siphon-rim closure as a virtually isolated system. The musculature consists of a large number of long, narrow bands of various relative size, distributed round the body-wall in a dense tangle in which only a few bands stand out. The fibres anastomose considerably, and end in fine branches which are embedded in the connective tissue of the bodywall. These muscles grade into those surrounding the siphons. The customary sharp distinction of the musculature into longitudinal and annular (Berrill, I950) is hardly justified in Phallusia and Ascidiella where the siphon muscles appear to be just an extension of the body-wall muscle complex which is strongly concentrated in these regions so as to surround the siphons. The 'annular' muscles, principally those round the siphons, have discrete, branching ends. There are strong bands attached to the siphon rims between the lappets. Contraction of these muscles and of others attached to them brings the siphon rim inwards and downwards towards the body, the rim 
being neatly folded so that complete closure results. There are few siphonencircling muscles in close proximity to the rim, but there is a strong band with its centre about $\mathrm{I} \mathrm{cm}$. distant from the rim.

When the longitudinal musculature is cut near the rim on one side, that side of the rim no longer contracts, and complete siphon closure is then impossible. Cuts in the encircling muscle band affect the contraction only slightly. Hence it is inferred that the principal muscles concerned with siphonrim closure are those which work at right angles to the rim. The encircling muscles control the diameter of the whole siphon cylinder, but principally serve to give the siphons rigidity and to provide attachment for other muscles.

Ascidiella is found in the same estuarine habitat as Phallusia. Average specimens are about $9 \times 4 \mathrm{~cm}$. The test is $3-6 \mathrm{~mm}$. thick. Its general organization is similar to that of Phallusia.

The experiments to be reported involve the use of whole animals and are therefore subject to all the difficulties encountered when trying to investigate phenomena which are undoubtedly complex and affected by a variety of factors, largely internal to the animal, which are not subject to experimental control. Nevertheless, as a preliminary to the investigation of isolated physiological units, which may prove possible in Phallusia, it is hoped that given adequate control, useful information may be derived from such a study.

\section{Methods of Recording and Experimental Procedure}

Animals to be investigated were allowed to rest in their natural position on a heavy glass plate, usually being secured lightly with cotton tape. The plate was placed in a large, heavy, porcelain tank or enamel basin capable of holding several gallons of sea water. These, in turn, were supported on tables built into a concrete floor. Kymograph motors were mounted on sponge-rubber strips. Under these conditions the animals were sufficiently isolated from vibrations. The recording system consisted of long levers of dried grass writing on smoked drums. The writing points were of thin glass tubing made up to give points of the frontal type mounted at right angles to the levers (Fig. I). Whenever the natural position of the animal permitted, a glass arm attached to the lever was allowed to touch the inside of the siphon rim, thereby transmitting movements to the lever. Alternatively, a small glass hook was bent to fit over, but not penetrate, the siphon rim, and this was connected by thread to the lever. The weight required to balance the levers when placed in the position normally occupied by the siphon rim was about $0.4 \mathrm{~g}$. so work done against the lever system was small with either method and the animals were not unduly disturbed by either the contact arm or the hook.

Stimuli were derived from condenser discharges. They were applied through silver/silver chloride electrodes contained in glass tubes filled with sea water. 
A potentiometer was connected across the stimulator terminals. The frequency of discharge was controlled by a metronome operating a post-office relay which also acted as stimulus marker. The electrodes were applied to the outside of the test in various positions.

With this method of stimulation results were, at first, extremely variable. During the investigations it became probable that this variation is connected in some way with the changing states of the animals associated with their
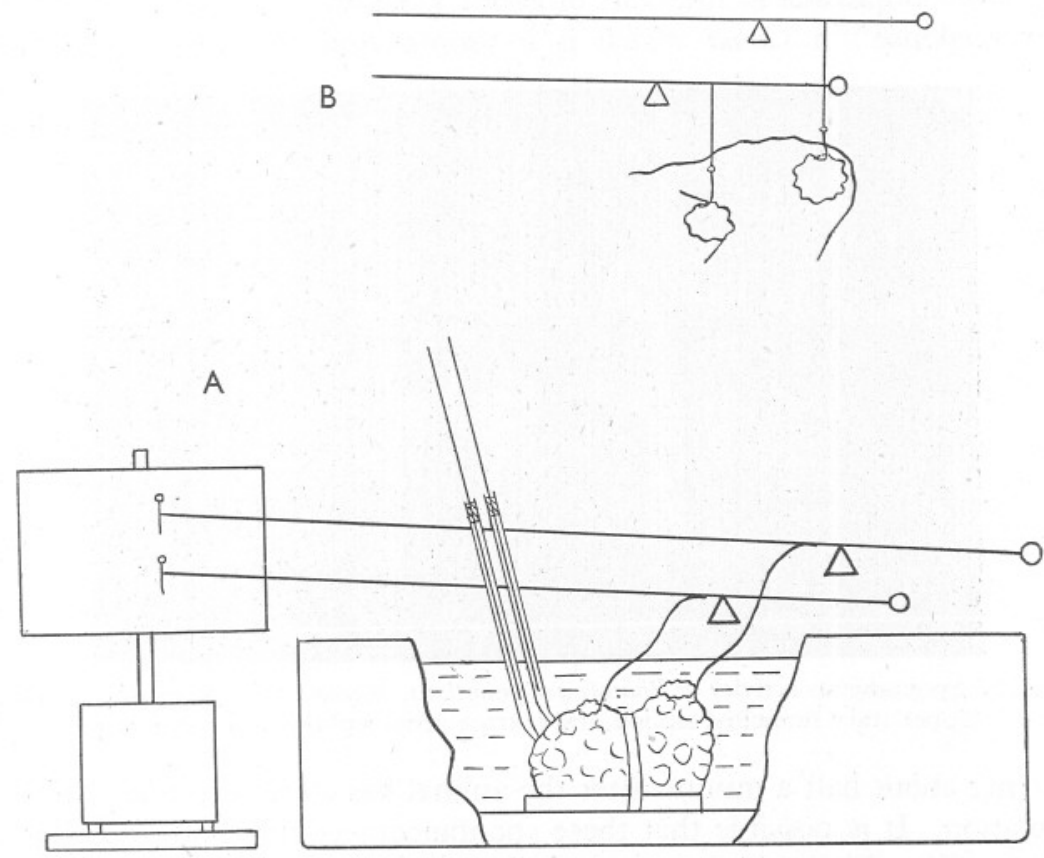

Fig. I. Diagram of method of recording and manner of stimulation showing use of:

(A) fixed glass arms; (B) glass hooks.

rhythmicity, and with the possible effect which the shocks might have on these states. Hence in Phallusia various experiments have been executed in an attempt to obtain an understanding of the interactions of background internal phenomena and external stimuli. The spontaneous activity shows a variety of patterns which will be discussed in another paper. The commonest pattern, that of synchronous activity of the two siphons at fairly regular intervals of 6-9 min. and continuing without interruption for several hours, is illustrated in Fig. 2. Other types of spontaneous activity may be related to external factors (cf. Batham \& Pantin, 1950). In order to be able to plan experiments which will give consistent and strictly comparable results it is important to be able to distinguish two sources of variation. These are: $(a)$ the variation in 
response to stimulation purely as a function of the stage in the spontaneous activity cycle which the animal has reached; $(b)$ the response to stimulation as a function of the previous history of the animal. It would be expected that (a) would show an additional variation with different test animals; $(b)$ would vary likewise but probably less than $(a)$.

The first type of variation is found to have two main components. Stimuli given immediately after a spontaneous contraction disturb the rhythmicity and frequently give rise to one or a series of small spontaneous contractions of variable amplitude at intervals of about $\frac{1}{2}-\mathrm{I}$ min. Kinoshita describes an 'after response' in Ciona which is a spontaneous incomplete contraction



Fig. 2. Spontaneous activity of Phallusia mammillata. Kecord reads from right to left. Upper trace branchial siphon, lower trace atrial siphon. Time in hours.

occurring about half a minute after the animal has started to relax following stimulation. It is possible that these spontaneous contractions (see Fig. I2) represent Kinoshita's 'after response'. Stimuli given just before a spontaneous contraction is due are abnormally effective in eliciting responses, for single stimuli may elicit a squirt at this time. This is not the normal reaction to a single shock, as will be shown below. These results illustrate the extremes of the first component of type (a) variation which is variation during the interval between individual squirts. The second component of type (a) variation is a much slower variation which is revealed by giving pairs of shocks at I min. intervals over a period of several hours. Although the stimuli are kept at $20 \%$ above the initial threshold there are frequent phases, each lasting for several minutes, when these stimuli give only a small response or even none at all. This observation is in line with the variations observed by Polimanti. Fig. 3 shows the effect of stimulating a specimen of Phallusia in this way. At the start of the series illustrated the shocks were ineffective. Spontaneous activity was accelerated and then the animal entered a phase which lasted 
for $20 \mathrm{~min}$. when the stimuli were equally effective. This phase ended almost as sharply as it began, apparently without further affecting the spontaneous activity. Later, with no increase in stimulus intensity a second period of responsiveness was apparent. Now, however, the response was weaker and more erratic. These results illustrate also the type $(b)$ variation referred to. The smaller size and erratic nature of the second set of responses shows the exhausting effect of the earlier stimulation.

Stimulation experiments must, therefore, be planned to avoid coinciding with the immediate pre- and the immediate post-spontaneous contraction period. They must also be conducted during phases of normal responsiveness, and they must not be executed too soon after a previous experiment. After stimulation several minutes may elapse before the same pattern of response can be re-elicited. The normal state can usually be said to be restored when the animal returns to its normal pattern of spontaneous activity.

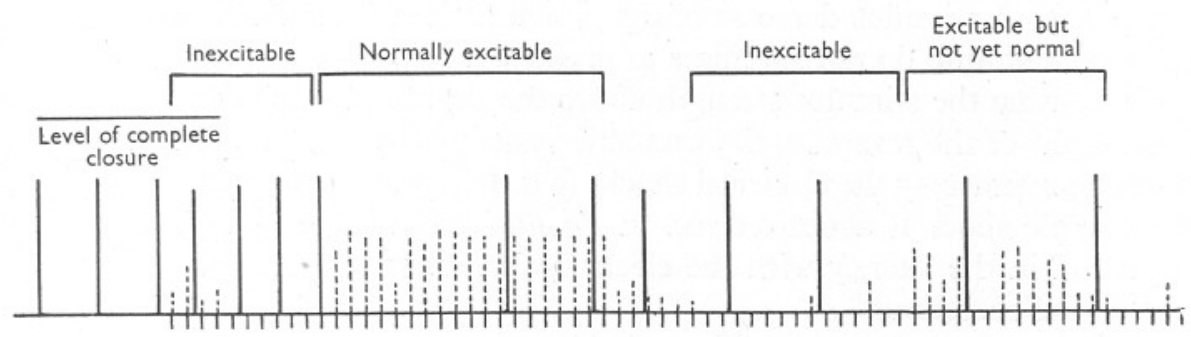

Fig. 3. The results of part of an experiment on the relation between excitability and internal phasic activity. The unbroken lines indicate spontaneous contractions, the broken lines indicate responses to electrical shocks. The marks below the baseline indicate the application of closely spaced pairs of shocks at I min. intervals between pairs. Phallusia, branchial siphon.

The experimental procedure used is therefore as follows. Only healthy specimens showing rapid movements of the siphons are used. Two equal test shocks I sec. apart and of suitable strength are given to the animal about $2 \mathrm{~min}$. after a spontaneous contraction. If the animal gives a good response a further minute is allowed to pass and then an experiment is carried out. Immediately after the experiment the animal is allowed to rest for IO-I $5 \mathrm{~min}$., or longer according to the length of the experiment, so that normal rhythmicity is restored. The procedure is then repeated. In about $5 \%$ of such tests the response is small or, rarely, absent, and a pair of shocks does not elicit a squirt (vide above types of variation). After a few minutes the response has always been normal again, further indicating that occasional phases of unresponsiveness are a normal feature of ascidian behaviour. 


\section{RESULTS OF EXPERIMENTS}

\section{Stimulus Strength}

The electrodes, separated by about half an inch, were placed in various positions on the test. The battery voltage and potentiometer were roughly adjusted until a pair of shocks gave a response. The potentiometer was then adjusted more carefully. The minimum strength required to produce a response varies with the position of the electrodes and the size of the animals. It is least when near the siphons. The strength required is usually large in Phallusia, of the order of $100 \mathrm{~V}$. from an $8 \mu \mathrm{F}$. condenser across most of a $50 \mathrm{~K}$. potentiometer. These facts are to be expected in view of the variable thickness of the test and the extent of short-circuiting of the shock. In Ascidiella the strength required is only about one quarter of the Phallusia value. The magnitude of the response to threshold stimulation does not vary with the position of the electrodes. At the least effective stimulation strength every stimulus is not always effective (Fig. 8a). A stimulus decrease of $0.5 \%$ makes the stimuli ineffective. Subthreshold stimuli do not summate to produce a response.

Increasing the stimulus strength above the threshold value does not affect the height of the response, the excitable system being activated in an all-ornothing manner by the electrical shocks (e.g. Fig. 6). The latency of response to a single shock is about $0.6 \mathrm{sec}$. in Phallusia, and there is no appreciable variation in the latency with the electrodes in different positions using this method of recording.

\section{The excitable system}

It is not possible at present to decide what the excitable system is, although it is clear from the results of the experiments on Phallusia and Ascidiella that during electrical stimulation the muscles receive the stimuli through a rapidly conducting system which is excited in an all-or-nothing manner by the stimuli. Von Buddenbrock (1928) stated that in ascidians there is a nerve network in the body-wall, but this statement has since been strongly contested by Fedele (1937a). Das (1936) has described nervous elements in the test of Herdmania. It is possible that local stimulation of the test is brought about by these elements. Whatever the excitable system may be, the situation is remarkably similar to that found in coelenterates, especially actinians, when these are stimulated in the same way (cf. Pantin, I935a-c, and Bullock, I943).

\section{Facilitation}

Many results are, nevertheless, quite different from those given by sea anemones. A single stimulus produces a measurable response, although this is small (Fig. 4) and occasionally ineffective (Fig. 8b). The second of a pair of shocks at intervals of I sec. down to about $0.3 \mathrm{sec}$. (the smallest interval used) produces a large, fast response (Figs. 4-6). Quantitative measures of the size of this response are not of great value, but it may be stated that over this range 
of interval between shocks there is no significant variation in the magnitude of the response (Fig. $7 a, b$ ). When the first stimulus is ineffective the second

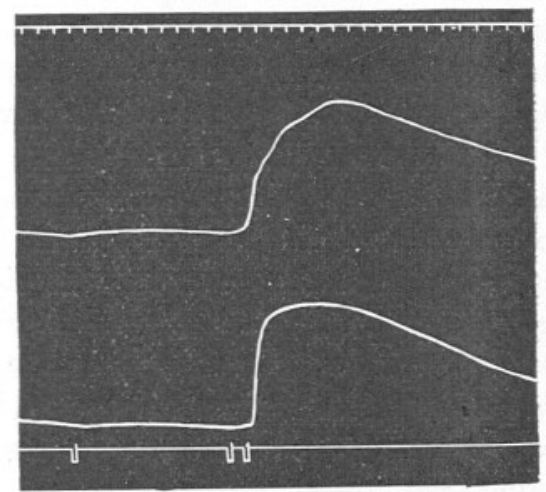

Fig. 4. Responses to a single shock and to a pair of shocks at I sec. interval. Phallusia. Upper record atrial siphon, lower record branchial siphon.

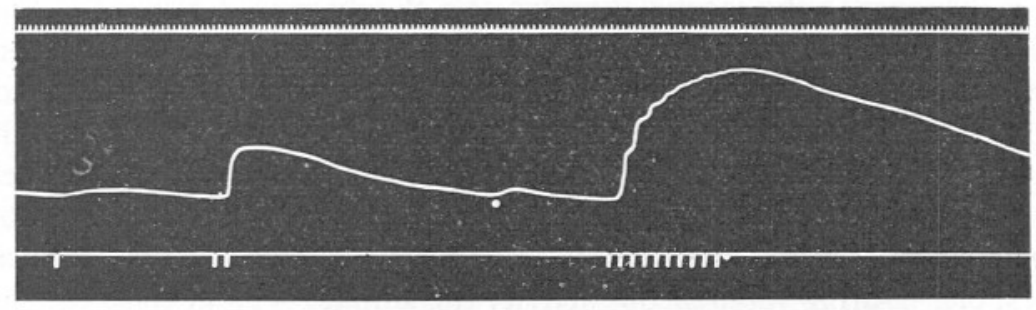

Fig. 5. Responses to a single shock, to a pair of shocks and to a consecutive series of ten shocks. Ascidiella. Branchial siphon. Time in sec.

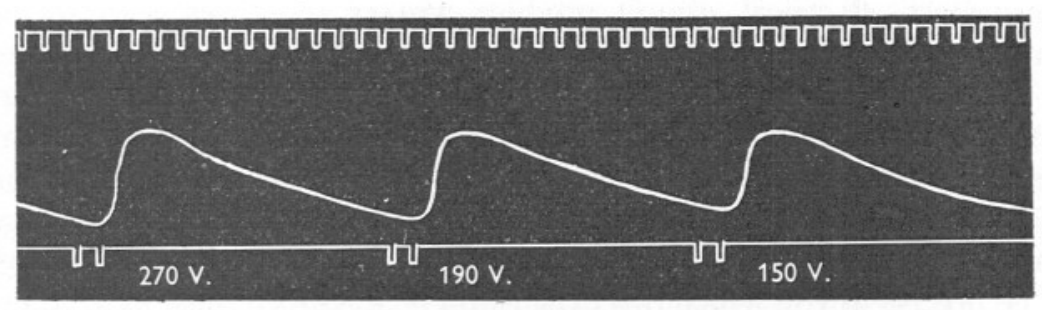

Fig. 6. Responses to pairs of stimuli at various voltages. Threshold was $145 \mathrm{~V}$. Phallusia. Branchial siphon. Time in sec.

may act like a normal first and the large response is then given to the third (Fig. 8b). Occasionally, three or even four stimuli are necessary before a first effective stimulus appears. 
The second of a pair of shocks in rapid succession produces a response which is so much larger than the response to the first that it looks as though a different effector mechanism has been brought into play. Lengthening the interval between a pair of shocks shows, however, that this large response is simply due to an extensive facilitation consequent upon the arrival of a first effective stimulus. At frequencies of I per $2 \mathrm{sec}$. and lower the height of the response to a pair of stimuli is reduced (Fig. $7 c$ ). There is still an enhancing effect of the first stimulus at a frequency of only I per IO sec. (Fig. $7 d$ ). The state of facilitation develops rapidly, as in anemones, stays at its maximum for I.5-2 sec. and then gradually declines, becoming negligible after IO-I $5 \mathrm{sec}$. After a closely spaced pair of shocks the aperture is almost half-closed and subsequent shocks produce contractions which serve to close it more completely. Further stimuli after the first two produce a typical smooth-muscletype staircase. The steepness of the staircase increases with increasing frequency of stimulation (Fig. $7 a-d$ ).

\section{Functional significance}

The functional significance of this type of response is fairly clear. The rapid rise in response to the second stimulus of a closely spaced pair is the characteristic squirt of the ascidians. The first stimulus simply closes the aperture slightly, the usual response to a slight mechanical vibration or light touch of the siphon. The second, effected by stronger vibration or contact, results in a rapid ejection of water through the closing aperture. This 'ejection reflex' (Jordan, 1908) has frequently been described as being of use in removing sources of irritation from the branchial cavity, ejecting sexual products or faeces, etc. During spontaneous activity the animals seldom close the siphon much further than the closure produced by two or three shocks in rapid succession. Complete closure only follows persistent stimulation. When the animal has recently been stimulated, or during periods of natural unresponsiveness, electrical stimuli produce weaker responses with increased latency and slower rate of rise of contraction (Fig. I4, and cf. also Kinoshita and Polimanti). These consequences, which are apparent after only one burst of electrical stimuli and increase progressively with further bursts until the animal is relaxed but inexcitable, are consistent with the view that the vigorous normal enhancement of the response to the second effective stimulus is due to an extreme development of facilitation, and that this mechanism is easily exhausted.

The precise way in which this exhaustion is developed must depend on the nature of the facilitation phenomenon. The indirect evidence points to the neuromuscular junction as being the site of facilitation in sea anemones. It is unlikely that direct evidence will be forthcoming. In the absence of the latter it is probably unwise to speculate, but if a junction transmitter is involved, exhaustion could be due either to its progressive depletion, a phenomenon which is not found in any known systems, or to its accumulation which is also 


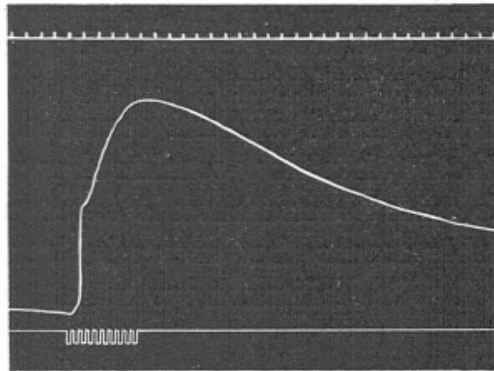

(a)

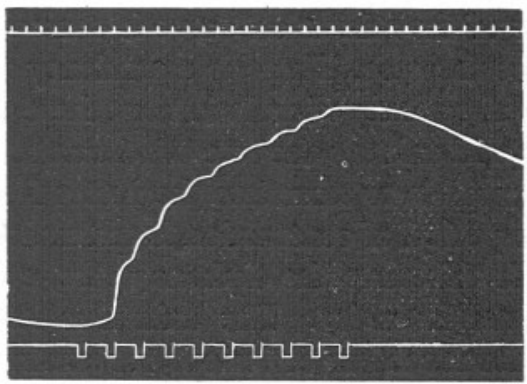

(c)

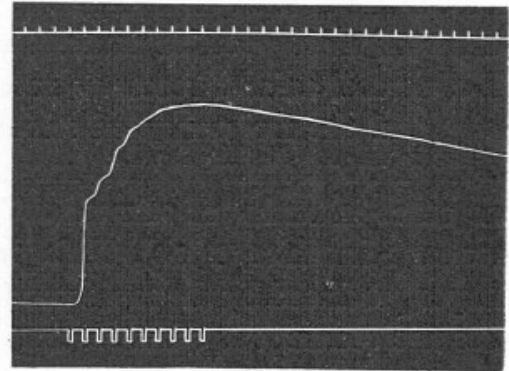

(b)

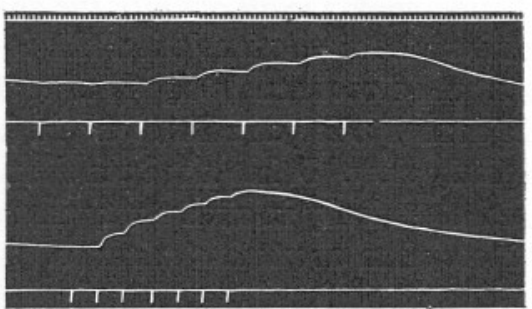

(d)

Fig. 7. Responses to: (a) 2 per sec.; (b) I per sec.; (c) I per 2 sec.; (d) I per 5 sec. (lower record); I per Io sec. (upper record). Phallusia. Branchial siphon. Time in sec.

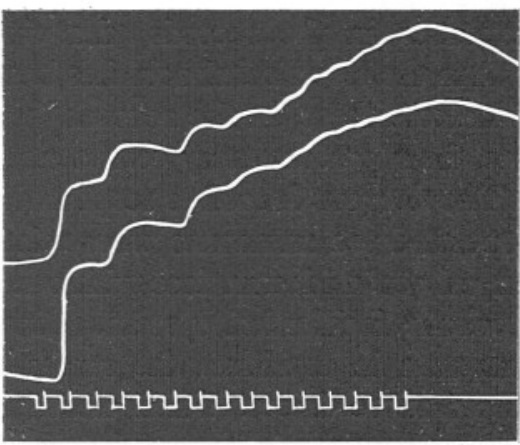

(a)

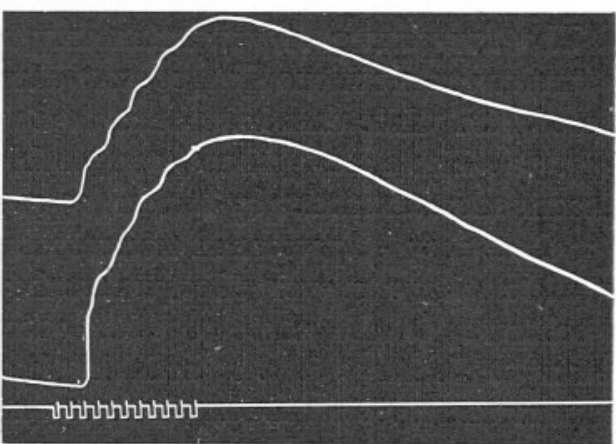

(b)

Fig. 8. Parallel behaviour of the atrial (upper record) and branchial (lower record) siphons showing also: (a) responses to just threshold stimulation at I per sec., not every stimulus is effective; $(b)$ ineffective first stimulus with delayed response to the second and quick response to the third. Phallusia. 
unlikely after such brief stimulation. An alternative possibility is that the phenomena are at least partly intramuscular in origin. Investigations into the properties of these peculiar muscles would be of great interest. The way in which a normal animal reacts to mechanical stimulation depends upon the intensity and the frequency of stimulation. Thus Polimanti observed that 'rapid mechanical stimulation is more effective than slow continued pressure for the same intensity of stimulus'. The same phenomenon is observed in anemones (Kinoshita, I9II). This probably means that successive sensory discharges produced by the rapid prods bring the facilitation system into play, whereas the continuous stimulus may not.

\section{Rate of Relaxation}

During spontaneous activity the shape of the relaxation curve is constant, but after electrical stimulation this is not the case. There is a tendency for relaxation to occur more slowly after slow frequency stimulation but there are exceptions to this generalization. In fatigued animals the rate of relaxation is extremely slow. It has not been found possible to stimulate the intact fresh animal electrically in such a way that the period of contraction is maintained for more than about five minutes. After this time the siphons relax, even though stimulation is continued at greater intensity. The animal is itself capable of maintaining the siphons closed for much longer periods, for example, following a period of exposure. These observations, together with others mentioned below in connexion with ganglion extirpation, suggest that relaxation is an active process in ascidians.

\section{Supernumerary Responses}

The appearance of supernumerary responses in the records, denoting stimulus contributions from sources other than the electrical shocks has been noted, and white dots have been used to indicate these on the records (e.g. Fig. 9c). These are, perhaps, comparable to the supernumerary contractions described in anemones by Pantin (I935c). The nervous impulses responsible for these may be present in normal unstimulated sea anemones where they would not have an overt effect unless they nearly coincided in time with other discharges, so that these together operated the facilitation system. Since a single discharge is a recordable phenomenon in ascidians the study of supernumerary contractions is there rendered more direct. When electrical stimuli are applied after a small spontaneous movement it is seen that these are effective in enhancing the response to subsequent shocks (Fig. 9c,d). Rhythmic supernumerary responses have also been observed, as mentioned above (p. 292) (see Fig. I2), although these occur at much longer intervals than those described in anemones. It seems highly probable that the events giving rise to supernumerary contractions are not a consequence of the electrical stimuli but arise independently of them, except in the case of the rhythmical 
contractions which may be due in ascidians to an effect of the shocks in changing momentarily the frequency of spontaneous activity.

\section{Ganglion Extirpation}

Experiments have been carried out on deganglionated animals. These animals usually respond to electrical stimulation like slightly fatigued intact animals, but there is no great difference in the pattern of response (Fig. II). Jordan (I908) and Hecht (I9I8) have studied the effect of ganglion extirpation on the responses of the siphons to mechanical contact. Before extirpation a slight mechanical stimulus to the outside of a siphon rim causes a small local

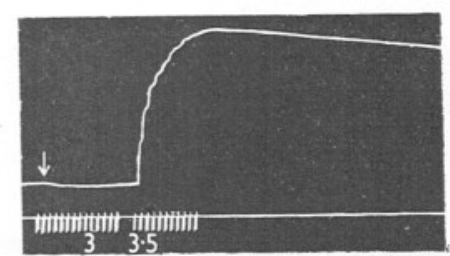

(a)

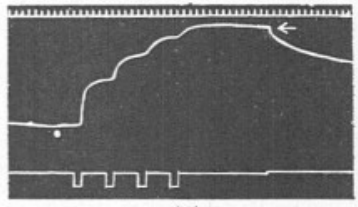

(c)

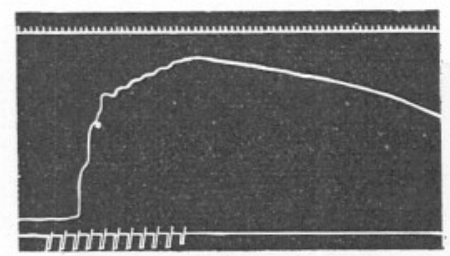

(b)

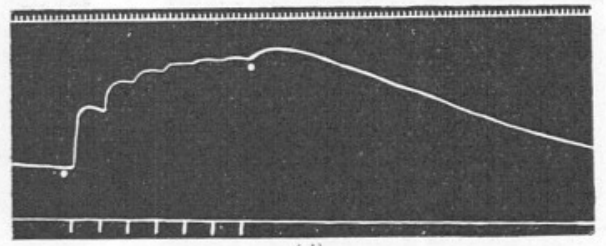

(d)

Fig. 9. Spontaneous movements and their consequences. Ascidiella. (a) Slight relaxation during subthreshold stimulation; (b) spontaneous movement (indicated by white dot) during stimulation experiment; $(c)$ stimulus applied $2 \mathrm{sec}$. after a spontaneous movement. Drum stopped at arrow; $(d)$ stimulus applied I sec. after a spontaneous movement.

response of the siphon. A larger stimulus causes closure of the other siphon as well as closure of the stimulated one. After ganglion extirpation the unstimulated siphon fails to respond, although the stimulated one continues to respond. Jordan described these responses as reflexes and regarded the second response as a protective reflex. If we confine the use of the term reflex to its original usage in physiology (as 'animal spirits' ascending nerves to central systems and in turn descending to effect motor activity) then these activities are better described as responses than as 'reflexes'. These responses can be studied graphically with this preparation (Fig. Io). In Phallusia it will be noted that in spite of the absence of the ganglion there is a slight response of the non-stimulated siphon when a single siphon is touched with a glass rod. When the containing vessel is tapped both siphons respond together. Day (I9I9) and Bacq (I935) agreed that the operation of ganglion extirpation reduces the general muscle tone. The fact that ganglion extirpation only 


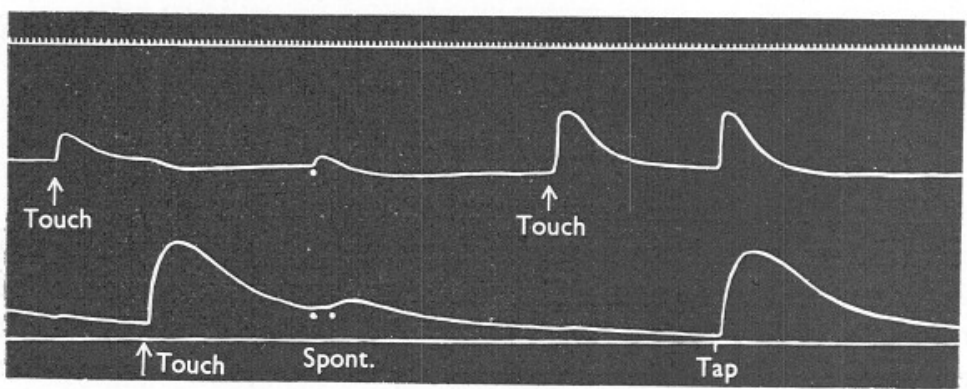

Fig. Io. Responses of a deganglionated Phallusia to local stimulation of the siphons and to a tap on the tank. Note the small response of the opposite siphon. Time in sec.

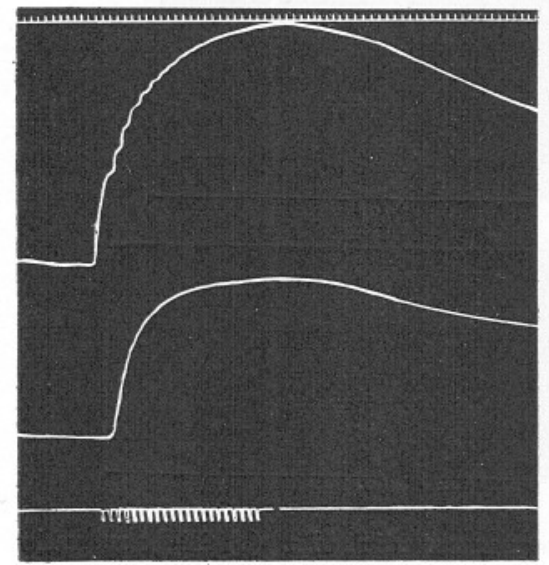

Fig. II.

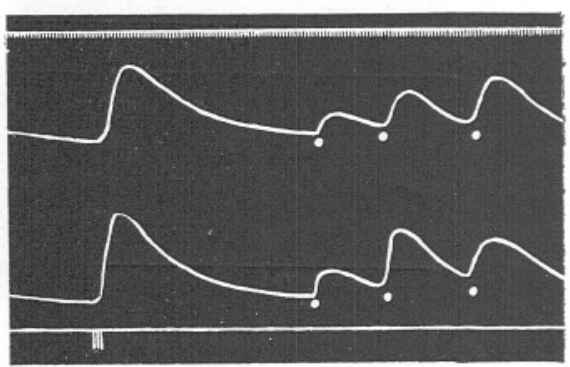

Fig. I2.

Fig. Ir. Responses of a deganglionated Phallusia to electrical stimulation at I per sec. Upper record branchial siphon, lower record astrial siphon. Time in sec.

Fig. I2. Spontaneous rhythmical contractions following an electrical stimulus (three shocks) applied soon after a spontaneous squirt (not shown). Phallusia. Time in sec.
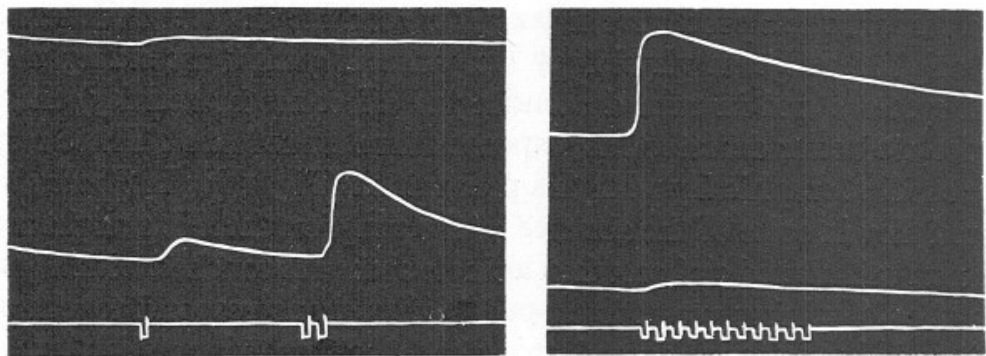

Fig. I3. Inhibition of one siphon only during stimulation experiments. Upper record atrial, lower record branchial in both records. Phallusia. Time in sec. 
affects the pattern of response to electrical stimulation indirectly, by the effect which this operation appears to have on muscle tone, shows that augmentation by the ganglion is not normally involved in the squirting movements. Also, the results show that the electrical stimuli are not transmitted to the muscles by way of the ganglion.

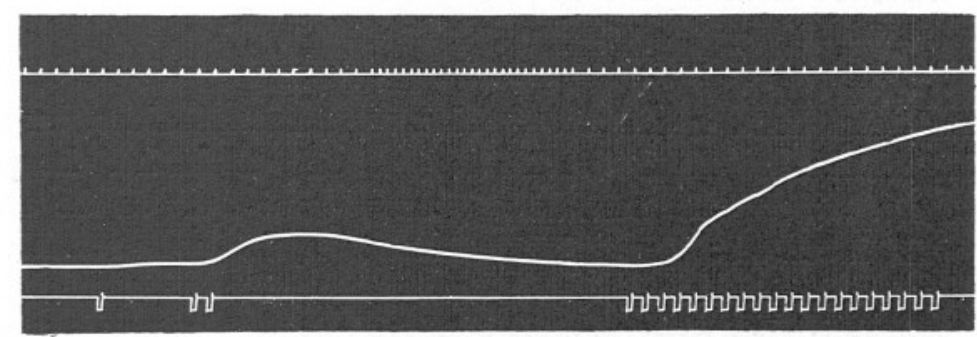

Fig. I4. Responses of Phallusia branchial siphon immediately following relaxation after intense'stimulation before the experiment. Time in sec. The drum was slowed down in the middle of the record to include the whole of the relaxation after the second response.

\section{Synchrony of the Siphons}

When responses are recorded from both siphons simultaneously, it is seen that these act in unison. Water is ejected through both siphons simultaneously through the closing apertures. The same water movements occur during spontaneous contractions which are also synchronous. Very rarely during stimulation one siphon fails to respond. In Fig. I3 the failure of one siphon has in turn been followed by inhibition of the other siphon after this had started to contract. This failure of the siphons to act together has not been observed in deganglionated animals during electrical stimulation. Jordan and Polimanti were both of the opinion that the ganglion can regulate the 'reflexes' by either inhibition or facilitation. Occasionally, small positive openings can be seen in the records (e.g. Fig. 9a) which correspond to reflexing movements of the siphon rims. It is difficult to see how these movements can be brought about except by quick positive relaxation. Similar movements can be observed in some of Yamaguchi's records. These observations strongly suggest that the ganglion can maintain or reduce tone in the muscles, and that it can prevent stimuli from giving effective responses.

\section{Test-free Animal}

When the animal has had its test removed it remains in a state of contraction for several minutes and then relaxes gradually. When relaxed, spontaneous contractions appear at intervals of a few minutes, as in the intact animal. These contractions involve the whole of the muscle mass simultaneously, as far as the eye can judge. They can also be produced by stimulating the siphon rims mechanically but not by touching the body-wall. In the latter case, 
a ripple of contraction travels outwards from the point of stimulation. The extent of this travel depends on the strength of the stimulus. Loeb (I90I), and later Fedele $(1937 b)$, thought that the muscles of ascidians are capable of direct excitation, and that they are also capable of propagating this excitation. Bacq (1935) demonstrated slow ripples of contraction in the siphons of Ciona. There are certainly at least two different methods of transmission of stimuli in ascidians.

\section{Discussion}

Ascidians show a number of interesting physiological peculiarities. Investigation of ascidian nerve-muscle should not only help to further the general study of these systems, but should also throw some light on the adaptive changes which can take place in nerve-muscle physiology in relation to an animal's way of life. The sessile sea-squirts have free-living relatives and when adequate studies have been made of both forms a comparison will be possible. There is also a parallel between the sedentary, or free-living members of the Tunicata and the corresponding forms of the Coelenterata, the sea anemones and the jellyfish. It is now possible to carry the comparison between the sedentary forms a little further.

Although no homology of intimate mechanism is postulated, and detailed consideration must await the results of further investigations, it is remarkable that these phylogenetically unrelated groups should include sedentary representatives which have evolved independently what appears to be a similar basic method of producing a protective response from a simple muscle system. Metridium and Phallusia inhabit similar environments and are often found together. They both feed on small particles and depend on a ciliary current for both feeding and respiration. A large central cavity is in each concerned with these activities and there is a terminal opening into the cavity. Protection is important not so much against predators, although these have been described (see, for example, Yonge, I949), as against interference with the continuity of the feeding and respiratory mechanisms by foreign bodies entering therein. The quick contractions of the longitudinal muscles of Metridium withdraw the animal from the source of the stimulus and produce a small current of water which helps to drive it away. Later, if the stimulus persists, the entrance to the all-important cavity is closed. In large, sedentary ascidians the facilitated quick contraction is more effective in producing a protecting jet of water than the actinian system, but as in the actinian is followed later, if the stimulus persists, by further withdrawal and by closure of the aperture.

The utilization of a single effector system which has no antagonists, to produce slow or quick contractions according to the nature of the stimulation by the development of facilitation to an extremely high degree, is an efficaceous and very economical method. The systems which have been evolved by several more highly organized animals to produce comparable effects, by the use of multiple innervation, giant fibres, etc., are not necessarily more effective. 
The investigations described serve to make the variations in some of the results of previous workers, published and unpublished (personal communications), a little clearer. Ascidians have patterns of spontaneous activity which affect the properties of the nerve-muscle system so that similar stimuli may elicit different degrees of response in the same animal at different times. The pattern of spontaneous activity, and other properties of the nerve-muscle system are modified for some time after a period of stimulation. The extent of the modification is proportional to the physiological condition of the animal and the duration of stimulation.

There remain, unfortunately, large gaps in our knowledge of the phenomenon of facilitation as it appears in anemones and in ascidians. First, we do not know the nature of the excitable system (this applies especially to ascidians) and the way in which it is activated by electrical shocks; whether, for instance, it gives trains of impulses or a single impulse for each condenser discharge. Secondly, we do not know anything about the physical phenomena occurring in the muscles during stimulation. There are several suggestions that intramuscular factors are involved. Thirdly, we have no notion as to the chemical nature of the junction transmitter. None of these problems can be solved until isolated physiological units are available for investigation.

The work was done at the Plymouth Laboratory; to the Director and staff of which most grateful thanks are extended. The writer occupied the London University table during parts of the spring and autumn of I95I. Gratitude is also expressed to Miss P. Kott and Dr J. A. C. Nicol for helpful discussion during my stay at Plymouth, and to Prof. O. Lowenstein, Dr G. P. Wells and Prof. P. B. Medawar for carefully reading the draft and offering valuable cirticism.

\section{SUMMARY}

A method is described for investigating the reactions and spontaneous activity of two species of ascidian, Phallusia mammillata and Ascidiella aspersa, which does not disturb the animals unduly. The method can be used for obtaining graphic records of the so-called 'reflexes' of ascidians.

If due attention is paid to certain factors which affect the state of the animals, they can be made to respond to electrical stimuli applied to the outside of the test in a way which can be observed repeatedly in the same animal, in other animals of the same species and in at least two genera of ascidian.

Electrical stimuli applied to the test affect the excitable system of the whole animal in an all-or-nothing manner.

Response to graded electrical stimulation reveals a facilitation mechanism which gives enormous enhancement to the size and speed of response to the second of a close pair of effective stimuli. It is suggested that this mechanism 
is responsible for the production of the rapid squirt of the large sedentary ascidians.

A possible cause of the very rapid fatigue of ascidians to all kinds of stimulation is fatigue of the facilitation mechanism following a period of stimulation.

A parallel is drawn between the responses of Metridium and the responses of Phallusia and Ascidiella. The responses have some common features and the response mechanism appears to serve a similar purpose in the life of the animals.

\section{REFERENCES}

BACQ, Z. M., 1935. Observations physiologiques sur le cœur, les muscles et le systeme nerveux d'une Ascidie Ciona intestinalis. Arch. Int. Physiol. T. 40, pp. $357-73$.

Batham, E. J. \& Pantin, C. F. A., I950. Phases of activity in the sea anemone Metridium senile, and their relation to external stimuli. Fourn. Exp. Biol., Vol. 27, pp. 377-99.

BERRILL, N. J., I950. The Tunicata. Ray Society, London. 354 pp.

vON BUDDENBROCK, W., 1928. Grundriss der Vergleichenden Physiologie, Berlin. 830 pp.

Bullock, T. H., I943. Neuromuscular facilitation in Scyphomedusae. Fourn. Cell. Comp. Physiol., Vol. 22, pp. 25 I-72.

DAS, S. M., I936. On the structure and function of the ascidian test. Fourn. Morph., Vol. 59, pp. 539-60.

DAY, E. C., I9I9. The physiology of the nervous system of the tunicate. I. The relation of the nerve ganglion to sensory responses. Fourn. Exp. Zool., Vol. 28, pp. 307-35.

FEDELE, M., I937 $a$. Ancora sulla inesistenza di una rete nervosa perifica nei Tunicata. Rend. Acc. Lincei., Vol. 25, pp. 656-61.

- 1937b. Contrattilità ed eccitazione neurogena e miogena negli 'Ascidiacea'. Rend. Acc. Lincei., Vol. 26, pp. 3 I-7.

Hecht, S., I918. The physiology of Ascidia atra. Fourn. Exp. Zool., Vol. 25, pp. 229-99.

JORDAN, H., I908. Uber reflexarme Tiere. Ein Beitrag zur Vergleichende Physiologie des Zentralen Nervensystems, vornehmlich auf grund von versuche an Ciona intestinalis und Oktopoden. Zeitschr. für Allg. Physiol., Bd. 7, pp. 86-r35.

KINoshitA, T., I9I0. Uber den Einfluss mehrer aufeinander folgender wirksamer Reize auf den Ablauf der Reaktionsbewegungen bei Wirbellosen. I. Mitteilung. Versuche an Tunicaten. Pflüg. Arch. ges. Physiol., Bd. 134, pp. 50I-30.

- I9II. Uber den Einfluss mehrer aufeinander folgender wirksamer Reize auf den Ablauf der Reaktionsbewegungen bei Wirbellosen. II. Mitteilung. Versuche an Coelenteraten. Pflüg. Arch. ges. Physiol., Bd. I40, pp. I67-97.

Loeb, J., I901. Comparative Physiology of the Brain and Psychology. New York. $309 \mathrm{pp}$.

Pantin, C. F. A., I935a. The nerve-net of the Actinozoa. I. Facilitation. Fourn. Exp. Biol., Vol. I2, pp. I19-38.

- r $935 b$. The nerve-net of the Actinozoa. II. Plan of the nerve-net. Fourn. Exp. Biol., Vol. I2, pp. 139-55.

- I 935 c. The nerve-net of the Actinozoa. III. Polarity and after-discharge. fourn. Exp. Biol., Vol. I2, pp. 156-64. 
Polimanti, O., I9I I. Beiträge zur Physiologie des Nervensystems und der Bewegung bei den niederen Thieren. II. Ciona intestinalis L. Arch. Anat. Physiol., Phys. Abth. Suppl. I9II, pp. 39-152.

YAMAGUCHI, Z., I93I. Some notes on the physiology of Styela clava. Sci. Rep. Tohoko Imp. Univ., Vol. 6, pp. 597-607.

Yonge, C. M., I949. The Sea Shore. London. 3 II pp. 